\title{
Spontaneous melodic productions of expert musicians contain sequencing biases seen in language production
}

\author{
Roger E. Beaty ${ }^{\mathrm{a}, 1,2}$, Klaus Frieler ${ }^{\mathrm{b}, 1}$, Martin NorgaArd ${ }^{\mathrm{c}}$, Hannah Merseal $^{\mathrm{a}}$, Maryellen \\ C. MacDonald ${ }^{\mathrm{d}}$, AND DANiel J. Weiss ${ }^{\mathrm{a}}$

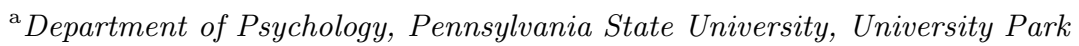 \\ ${ }^{\mathrm{b}}$ Institute for Musicology, University of Music Franz Liszt Weimar, Germany \\ ${ }^{\mathrm{c}}$ School of Music, Georgia State University, Atlanta \\ ${ }^{\mathrm{d}}$ Department of Psychology, University of Wisconsin-Madison, Madison \\ ${ }^{1}$ Authors contributed equally \\ ${ }^{2}$ Corresponding Author: Roger E. Beaty, E-mail: rebeaty@psu.edu
}

February 15, 2020

\begin{abstract}
Language production involves complex action sequencing to produce fluent speech in realtime, placing considerable constraints on working memory that lead to sequencing biases in production. Researchers have speculated that these biases may extend beyond language to other human behaviors involving action sequencing, but this claim has not been empirically investigated. Here we provide a strong test of this hypothesis, examining whether biases seen in language production also constrain one of the most complex and spontaneous human behaviors: musical improvisation. Using a large corpus of improvised solo transcriptions from eminent jazz musicians, we test for the existence of an established production bias observed in language production termed easy first - a tendency for more accessible sequences to occur at the beginning of a phrase, allowing incremental planning of more complex phrases. Our analysis shows consistent evidence of easy first in improvised music. We find that the beginning of improvised musical phrases contains 1) more frequently occurring interval patterns, 2) smaller intervals, 3) less interval variety, 4) less pitch variety, and 5) fewer direction changes. There was no easy first bias in a control corpus containing simulated data with the same structure, indicating that the effects are specific to real-time melodic production and not simply due to stylistic conventions. The findings indicate that even expert jazz musicians, known for spontaneous creative performance, reliably retrieve easily-accessed melodic sequences before creating more complex sequences - consistent with an incremental planning strategy employed in language production - suggesting that similar biases constrain the spontaneous production of music and language.
\end{abstract}

Charles Darwin famously observed that "the capacity of producing musical notes... must be ranked amongst the most mysterious with which (man) is endowed" [7]. While Darwin was concerned with understanding the adaptive significance of music, a fundamentally similar state of uncertainty also shrouded the cognitive underpinnings of music production. Insight into both of these issues would finally arrive 80 years later when Karl Lashley suggested that all complex sequential actions require hierarchical planning $[9,22]$. Thus, the underpinnings of both music and language production share common origins, both phylogenetic and cognitive, with hierarchical action plans. While this view has generally been endorsed [22], there has been virtually no evidence supporting the notion that common cognitive processes underlie the spontaneous production of music and language. Studying the potential link between language and music production may yield insights into how humans differ from other species. We begin to tackle this longstanding issue by investigating whether one of the cognitive biases known to influence word ordering choices in speech production also manifests to constrain sequencing in the context of spontaneous music production. 
Producing language requires the retrieval of words from long term memory that match the ideas the producer wants to express, sequencing those words into a sentence, and ordering the articulatory gestures that allow the words to be articulated [11]. Like all action, language production is guided by an internal hierarchical plan [22], and development of the plan is thought to be incremental, such that speakers begin executing the plan (articulating early parts of an utterance) while continuing to plan later parts [10]. Incremental planning is advantageous because it minimizes the amount of information that must be maintained in working memory until it can be executed. This incremental planning is most valuable when there is flexibility to order subcomponents that are ready to be articulated earlier in the plan, allowing for articulation to begin and providing more time to plan portions of the utterance that are not ready to be uttered. Languages have this flexibility, including ones like English with fairly rigid word orders, as most ideas can be expressed using many different words and word orders $[5,12]$.

Both language production experiments and analyses of spontaneous conversations show robust tendencies for speakers to utter easier words and phrases - more frequent, shorter, recently mentioned, or more salient-before harder ones, a bias termed accessibility or easy first $[2,12]$. For example, consonants that are easier to articulate in the infant vocal tract tend to precede more difficult ones in babbling sequences [13]. Several computational accounts of motor planning, task optimization, and cognitive control contain mechanisms that would tend to generate an easy first bias in serial ordering $[3,8]$, suggesting that these biases are an adaptive consequence of the need to sequence behaviors in any incremental action system. Nevertheless, empirical evidence for the existence of domain-general sequencing biases is scarce outside of language production research.

Here we aim to provide a strong test of the hypothesis that sequencing biases seen in language production can also constrain one of the most complex spontaneous human actions: musical improvisation. We analyzed a corpus of thousands of improvised sequences extracted from popular jazz recordings produced by eminent jazz musicians, an expert group defined by their spontaneous creative performance. We tested whether spontaneous improvisations follow the easy first bias observed in language production. To test for an easy first bias in music production, we examined multiple definitions of 'difficulty' analogous to those seen in language production, including 1) less frequently occurring interval patterns (i. e., sequences of pitch changes), 2) larger intervals, 3) more interval variety, 4) more pitch variety, and 5) more pitch direction changes. This approach allowed us to examine whether production biases consistently seen in language can also explain complex creative behavior in music.

\section{Results}

We extracted all melodic sequences (i. e., "n-grams"; length 3 to 10 intervals) and corresponding phrase information (i.e., n-gram positions within phrases) from the Weimar Jazz Database (WJD), a collection of 456 monophonic solos from 78 well-known jazz musicians (e.g., Miles Davis, Charlie Parker, John Coltrane) transcribed manually by expert transcribers and musicologists (Fig. 1; [18]). For the set of all extracted interval n-grams, we calculated both extrinsic and intrinsic difficulty measures. Extrinsic difficulty is based on the entire corpus; intrinsic difficulty captures features of a pattern per se. Extrinsic difficulty was quantified by the negative logarithm of occurrence probability (i. e., surprisal), as estimated by the relative frequency of an n-gram (based on all n-grams of the same length). Thus, the less frequent the n-gram, the higher the extrinsic difficulty value. For intrinsic difficulty, we computed several measures that reflect different conceptualizations of difficulty (e.g., interval variety and interval range; see Method). Because some intrinsic difficulty measures were highly correlated with one another (SI Results), to simplify analyses and to obtain a more numerically stable measure, we computed a composite intrinsic difficulty measure for analysis, as the standardized sum of a subset of intrinsic difficulty measures (Method). To control for effects due to stylistic convention, and for possible statistical artefacts of the analysis process, we created a simulated corpus using first-order interval Markov Chains. This control corpus is exactly the same length and contains the same distribution of intervals, interval transitions, and phrase lengths as the actual WJD corpus, which is effectively a sophisticated scrambling of the order of tones in the original corpus. 


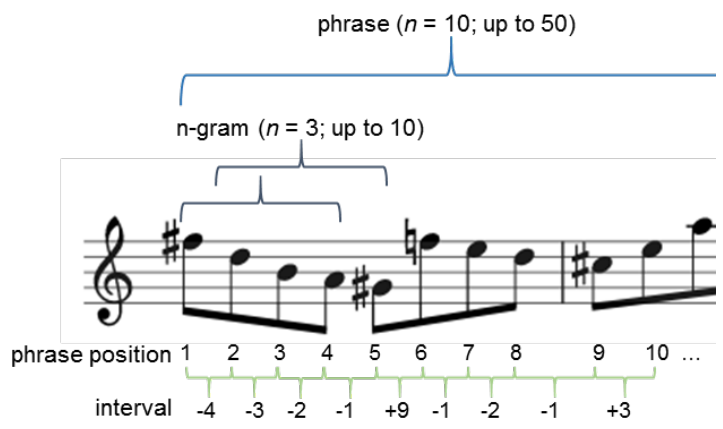

Figure 1: Example melodic improvisation phrase and corresponding metrics used for analysis. N-grams ( $n=3$, up to 10 notes) consist of interval patterns embedded within these longer phrases ( $n=10$; up to 30 notes). Phrase position provided temporal information to test the easy-first hypothesis.

\section{Correlations between intrinsic and extrinsic difficulty}

We began by computing correlations between the extrinsic and intrinsic measures of melodic difficulty to test whether the frequency of a sequence tracks its complexity. Results showed positive correlations between extrinsic and intrinsic difficulty: $r=.58$ for shorter sequences $(n=3)$ and $r=.30$ for longer sequences $(n=10)$. The same pattern of positive correlation was found between extrinsic difficulty and the individual difficulty measures (e.g., interval variety; SI Results). The simulated corpus showed similar but consistently lower correlations between extrinsic and intrinsic difficulty $(r=.54$ for $n=3$ and $r=.05$ for $n=10)$. Critically, the simulated corpus showed that the difficulty effect cannot be explained by a general preference for smaller (i. e., easier) intervals in improvised music (SI Results). The correlation analysis thus suggests that, overall, expert musicians tended to produce melodic sequences that were more frequent and less complex when improvising: as the frequency of melodic sequences in the corpus increased (extrinsic difficulty), the complexity of those sequences decreased (intrinsic difficulty).

\section{Easy first analysis}

Having demonstrated that easier melodic sequences tend to occur more often in improvised music, we turned to test the temporal question of when these sequences are likely to occur. The "easy first" hypothesis posits that sequence complexity should be lowest at the beginning of a phrase - defined here as the first 10 to 20 notes - which would allow incremental planning of more complex sequences at later phrase positions. First, we calculated Spearman's rank correlations between phrase position and difficulty. To visualize the effects, we created separate plots for n-gram lengths 3 to 10 (Fig. 2 \& Fig. 3), with phrase position on the X-axes and difficulty values on the $\mathrm{Y}$-axes. Inspection of these plots reveals consistent evidence of easy first, most prominently for intrinsic difficulty (Fig. 2). The plots suggested a nonlinear relation between difficulty and phrase position; this nonlinear relation was confirmed with polynomial regression analyses, which, across full and averaged data (mean values per phrase position), showed a consistently better fit to the data compared to linear models, with convex shapes that rise at the beginning of phrases (Fig. 4 \& SI Results). Due to the large data amount of data, all regression models were significant at $p<.001$.

Critically, analysis of the simulated data did not show easy first effects for both intrinsic (Fig. 2) and extrinsic difficulty Fig. 3). Slopes in the simulated data were largely flat and hovered around the midpoint of zero, providing evidence that the difficulty effects in the real data were not due to artefacts of the analysis or stylistic conventions of music. Two ANOVA models, with extrinsic and intrinsic easiness as dependent variables and data type (real or simulated) and phrase position as independent variables (n-grams with lengths 3 to 10 and phrase positions from 1 to 30 ) confirmed that these differences are significant (intrinsic difficulty: $R_{\text {adj }}^{2}=.18$, 

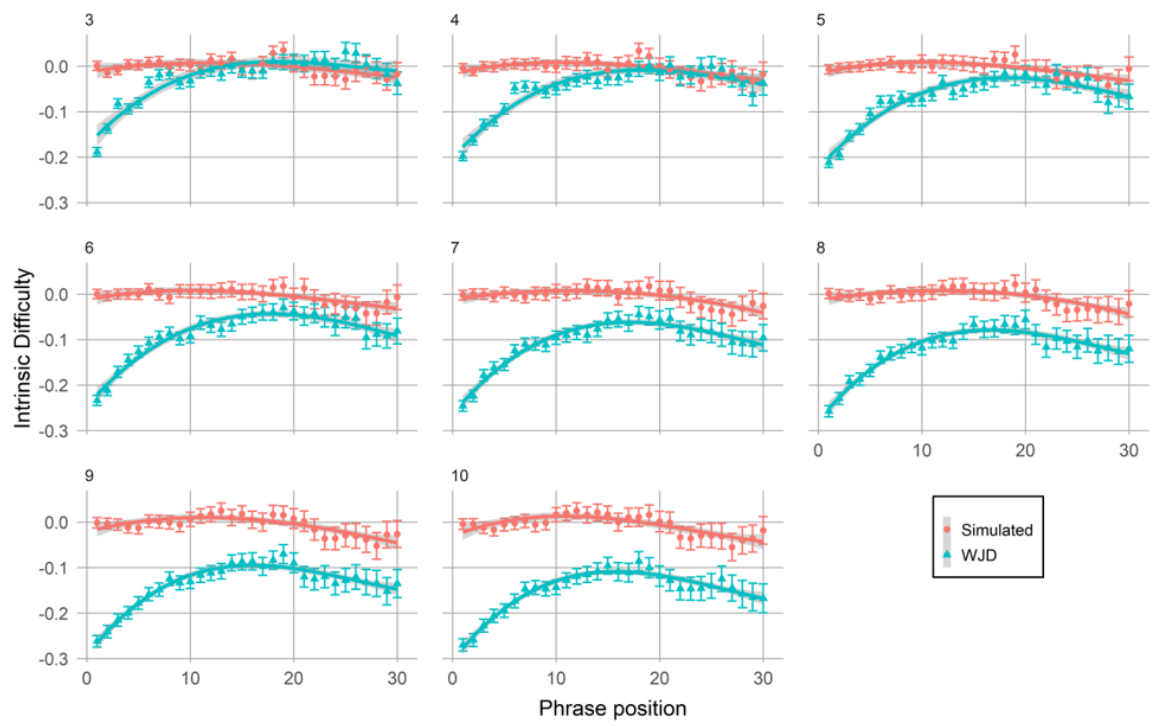

Figure 2: Plot of standardized intrinsic difficulty against phrase position in the improvisation corpus (WJD) and simulated corpus. In contrast to the simulated corpus, musicians tended to begin a phrase with easier (less complex) sequences and proceed to harder sequences later in a phrase. The eight panels correspond to the different sequence (n-gram) lengths (3 to 10). Error bars are standard error; curve fits are cubic polynomials. X-axis indicate phrase position of the first note of an n-gram and $\mathrm{Y}$-axis indicates combined intrinsic difficulty.

$F(2,477)=53.2, p<.001$, extrinsic difficulty: $\left.R_{\text {adj }}^{2}=.11, F(2,477)=30.25, p<.001\right)$. Regarding effect sizes, we focus on the range of difficulty values - the difference between maximum and minimum values - across the entire set of phrase positions, with range measured in standard deviation units akin to Cohen's $d$. Across all sequence lengths (3 to 10), we found that mean standardized difficulty values were negative (Fig. 2 \& Fig. 3), i. e., difficulty values at the beginning of the phrases were below the average of all sequences in the database, with values for intrinsic difficulty (and extrinsic difficulty) between $-.17(-.11)$ for shorter sequences $(N=3)$ and $-.10(-.01)$ for longer sequences $(N=10)$, indicating that easier melodic sequences were produced first. The effects were more pronounced for intrinsic difficulty (Fig. 2) than for extrinsic difficulty (Fig. 3), indicating that sequence complexity is more strongly linked to the difficulty bias than sequence frequency. Expert musicians thus reliably produced "easier" sequences before producing more complex sequences, consistent with the easy-first production bias observed in language.

\section{Discussion}

Language production is thought to be aided by first producing more easily accessible sequences before producing more complex sequences, a production bias known as easy first. Here we show that the easy first bias extends beyond language production to constrain high-level musical performance - musical improvisation, often considered one of the most complex creative behaviors. Large-scale corpus analysis of several hundred recordings from eminent jazz musicians revealed consistent evidence of an easy first bias in improvised music. We found that musicians consistently produced easier melodic sequences - defined both as more statistically frequent and less melodically complex - at the beginning of improvised phrases, with frequency and complexity gradually increasing with increasing phrase positions. Critically, simulated data with the same interval structure showed virtually no evidence of easy first, suggesting that the easiness effects reflect production biases for improvised music and not simply a tendency for smaller intervals or overall stylistic conventions of jazz music. Taken together, our results indicate that similar 

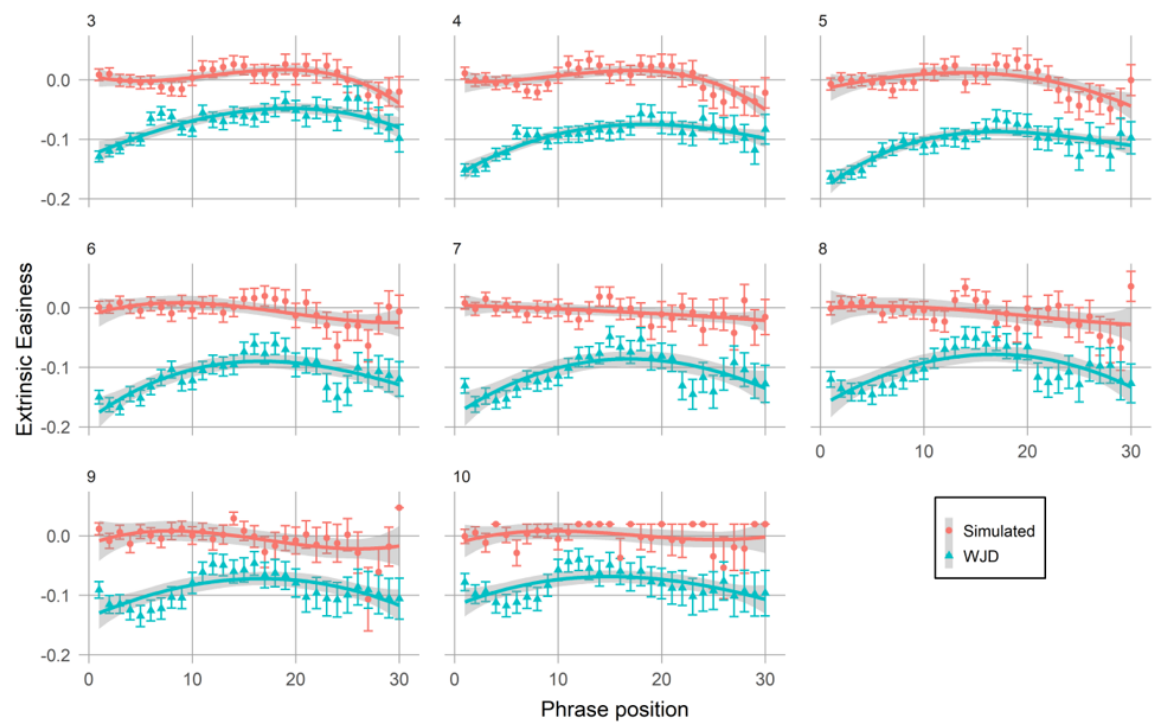

Figure 3: Plot of standardized extrinsic easiness against phrase position in the improvisation corpus (WJD) and simulated corpus. In contrast to the simulated corpus, musicians tended to begin a phrase with easier (more frequent) sequences and proceed to harder sequences later in a phrase. The eight panels correspond to the different sequence (n-gram) lengths (3 to 10). Error bars indicate standard error; curve fits are cubic polynomial. X-axis indicates phrase position of the first note of the n-gram and Y-axis indicates extrinsic difficulty, defined as the negative logarithm of occurrence probability (standardized) as compared to all phrases in the improvisation database.

production biases may constrain the spontaneous production of both language and music.

We presented evidence of a domain-general sequencing bias influencing spontaneous melodic production, which has largely been documented in language production ([12]; but see [14] for research examining music and language sequence execution). Sequencing biases, such as easy first, are thought to minimize the computational burden of language production via incremental planning. Beginning with more easily accessible words and phrases allows the speaker to simultaneously begin producing easy material and continue planning of more complex material, freeing working memory and allowing speech to initiate more quickly than if the complex material had to be planned first. Such temporal constraints similarly affect musical improvisation, which requires the real-time expression of aesthetically-appealing music with minimal time to plan $[19,20]$. In improvised jazz, musicians must meet the cognitive and physical constraints of performing fast and complex melodies. Having expert knowledge of common motor patterns (melodic sequences) can minimize these computational burdens [15, 16, 17, 19, 20], allowing the improviser to focus on other aspects of performance and plan more novel and interesting melodic sequences.

Given the novelty-driven aesthetic of jazz, it is remarkable that expert musicians reliably produced less novel (i. e., easier) melodic sequences. A general tendency for jazz musicians to avoid simplicity (and to strive for complexity) can be assumed, as this is a core feature of modern jazz aesthetics $[1,4]$. Although the effect sizes are small and the variances for phrase positions are large, the easy first effects are quite robust, suggesting that this production bias is indeed fundamental to the spontaneous production of music, at least in the context of tonal jazz. It may seem paradoxical that some of the most renowned jazz musicians in history (e.g., John Coltrane, Charlie Parker) insert quantifiably common (i. e., high-frequency) melodic patterns early in their phrases. Our study does not address musicians' motivations, but the adaptive character of incremental planning seems a likely factor, where easy patterns early on allow planning of more complex melodic patterns later in a phrase.

Our finding of easy first biases also extend past research on pattern analysis showing re- 

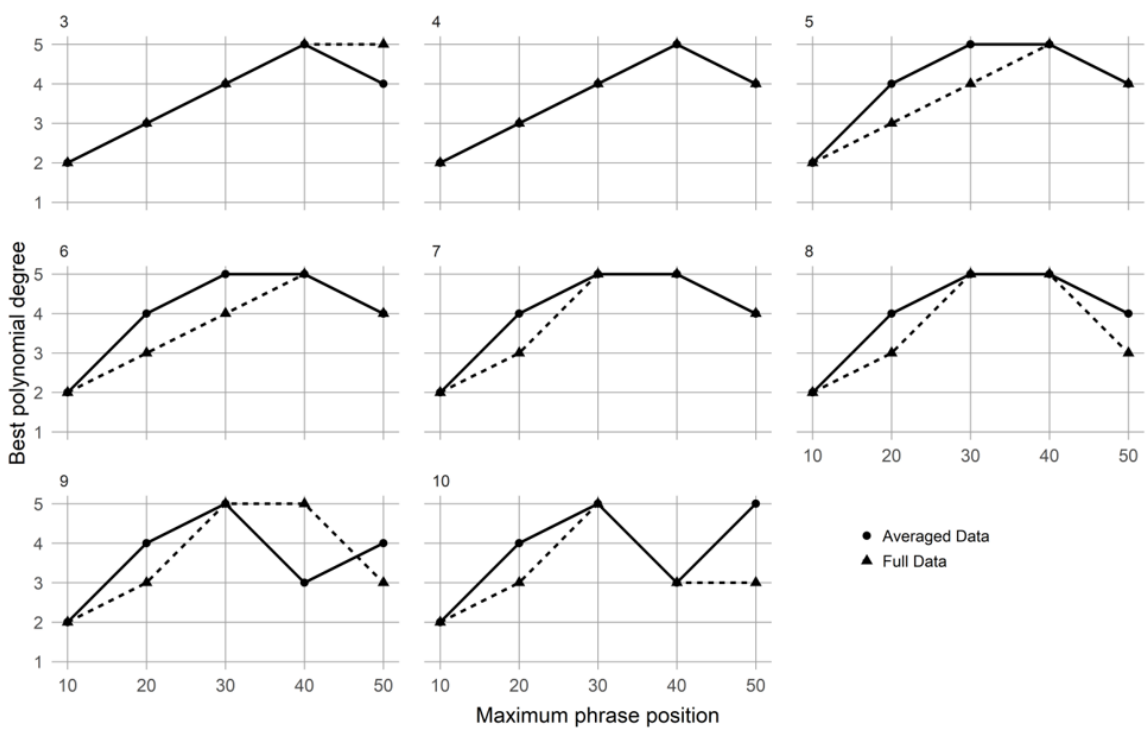

- Averaged Data

^ Full Data

Figure 4: Best fitting polynomial degrees (y-axis) of regressing intrinsic difficulty against phrase position. Analysis conducted separately for sequence orders between 3 and 10 (panels), maximum phrase position between 10 and 50 (x-axis), averaged (points, continuous lines; mean values per phrase position) and full data (triangle, dashed). All regression models have $p<.001$.

curring melodic sequences in improvised musical performance $[16,23]$ as well as an influential theory of improvisation emphasizing the importance of schemas in automating aspects of performance to meet the temporal, physical, and psychological constraints of improvisation [20]. The present work extends previous corpus-based analyses by considering overlapping patterns in melodic sequences, circumventing the problem of defining discrete melodic units (within continuous strings of notes) using arbitrary criteria [16]. We also extend previous corpus work by examining positions of n-grams within individual phrases, allowing the detection of fine-grained temporal trends in improvised performance. Taken together, the findings suggest that high-level creative performance is partly facilitated by domain-general sequencing biases that support language production, and that the substantial physical, temporal, and psychological constraints of spontaneous creativity can be mitigated by first producing less complex and easily accessible melodic sequences. Thus, an insight from the language production literature (i.e., easy first) reveals constraints on spontaneous musical creativity.

It is worth noting that easy first is not the only bias that shapes the serial order of words and phrases in language production. Other sequencing biases, including plan reuse, operate on the level of syntax, promoting repetition of higher-order linguistic structure [2, 12]. The present work establishes the existence of one sequencing bias in music production, but whether other known biases such as plan reuse influence melodic sequencing remains an open question. Another key question for future research concerns the role of production biases in shaping perception/comprehension. According to the Production-Distribution-Comprehension model, sequencing biases such as easy first shape language perception through the statistical regularities created by speakers following the biases: the more a listener perceives language with an easy first structure over their lifetime, the easier such structures will be to comprehend, leading to greater preferences for sequences with easy first ordering [12]. To what extent does such statistical learning influence musical preferences? One possibility is that, given the high frequency of the easy first pattern in music improvisation, expert musicians may develop an expectation of, and preference for, melodic sequences that follow easy first (and other sequencing biases).

More generally, our findings open the door for deeper investigations of other domains with respect to sequencing biases that align with linguistic biases, with potential implications for distinguishing uniquely human behaviors like music and language from behaviors found in other species. Sequencing biases such as easy first are well-documented in language [12], and re- 
searchers have begun to speculate about their relevance in other types of actions [21], including reaching and grasping, which appear in other species. To our knowledge, the current study is among the first empirical investigations testing the easy-first bias outside of language, and further work is needed to establish the scope of such biases. Sequencing biases may be limited to producing highly complex hierarchical structures like language and music; if so, they could be seen as a hallmark of uniquely human behaviors. Alternatively, sequencing biases may be part of a domain-general "syntax of action" [22], not limited to language and music. Further pursuit of the degree of sequencing commonalities in music and language, and in action more broadly, may thus inform theories of the components of human cognition.

\section{Material and Methods}

All data, analyses, and visualizations can be found in a supplementary online application: https: //jazzomat.hfm-weimar.de/easy_first/.

\section{Music database}

The Weimar Jazz Database (WJD) included solo transcriptions from recordings ranging from 1925 to 2009. The top performers with the most solos are John Coltrane (20 solos), Miles Davis (19 solos), Charlie Parker (17 solos), Sonny Rollins (13 solos), David Liebman (11 solos), Michael Brecker (10 solos), Wayne Shorter (10 solos), and Steve Coleman (10). Regarding jazz styles, the distribution is traditional jazz (32 solos), swing (66), bebop (56), cool jazz (54), hardbop (76), post-bop (147), and free jazz (5, only Ornette Coleman). Furthermore, the WJD contains 158 tenor saxophone solos, 101 trumpet solos, 80 alto saxophone solos, 20 trombone solos, 23 soprano saxophone solos, and 68 solos by other instrument (including a few piano and guitar solos). Note that the distribution of solos over performers and jazz styles is not uniform or random but reflects perceived importance of players and specific research interests of the creators of the database.

The solos were transcribed manually as pitch, onset, and duration tuples by expert transcribers using Sonic Visualiser [6] and carefully cross-checked several times to ensure a very high quality. The solos in the WJD are also annotated for a variety of features, such as beats, bars, metrical information, chords, and form parts. Phrases were annotated manually by the transcribers. For this study, we use only semitone intervals (difference between consecutive pitches) and phrase annotations. The WJD contains 200,809 tone events, which amounts to 200,353 intervals as intervals between tones in different sections were not counted. There are 11,802 phrases with a mean length of 18.1 (median $=13)$ tones $(\mathrm{SD}=15.4)$. The distribution of phrase lengths is heavily right-skewed (skewness $=2.5$ ) with a range from 1 to 203.

\section{Simulated data}

As a baseline, we simulated the 456 improvised solos in the WJD, matching the solo lengths. We used a first-order interval Markov model based on the WJD data, including the same phrase annotations from the WJD. The rationale behind this is to exclude effects from unusual (i.e., infrequent) interval distributions of jazz solos, which, like many Western melodic corpora (folk, classical, pop) has a strong preference for small intervals, i. e. semi-tone and whole-tones (but fewer tone repetitions than other corpora).

\section{Difficulty measures}

For the set of all extracted interval n-grams, we calculated a set of extrinsic and intrinsic difficulty measures. Extrinsic difficulty is based on the entire WJD corpus, whereas intrinsic difficulty captures features of a pattern per se. We used a single extrinsic difficulty measure that captured the negative logarithm of occurrence probability (i. e., surprisal), as estimated by relative frequency of an n-gram (based on all n-grams of the same length). Regarding intrinsic difficulty measures, we use the following putative observations to define an "easy" sequence: 
1. On average, larger intervals are harder to play and to perceive. For an n-gram interval $I$ with elements $\left\{i_{1}, i_{2}, \ldots, i_{n}\right\}$ this can be operationalized by either the mean or the maximum of absolute interval values, which yields two difficulty measures: max_abs_int $(I)=$ $\max _{k}\left|i_{k}\right|$ and mean_abs_int $(I)=\sum_{k}\left|i_{k}\right|$. The range of possible values is only bounded by the maximal possible absolute interval values (i. e., 127 for the MIDI pitch representation used in the WJD; note that the largest value in the actual data is 42 ).

2. A sequence should be more complex when it has a larger variety of intervals and a larger variety of corresponding pitches. This can be operationalized by the mean number of different intervals (int_variety) or pitches (pitch_variety). int_variety $(I)=\#\{I\} / n$, where $\{I\}$ is the set of different elements in $I$, and the number operator \# returns the number of elements in a set. pitch_variety $(I)=\frac{\#\{S I\}}{n+1}$, where $S I$ are the cumulative sums of $I$, $S I_{k}=\sum_{0 \leq j \leq k} i_{k}$, with $i_{0}=0$, which has $n+1$ elements. Both features have a value range between 0 and 1 .

3. An n-gram pattern should be more complex when it often changes directions. This can be operationalized by the mean number of directions changes (dir_change) or mean length of runs of an interval direction (mean_run_length). dir_change is simply the number of direction changes in a pattern, where a direction change can be measured by using the sign of the difference of interval signs:

$$
\operatorname{dir} \_ \text {change }(I)=\sum_{1 \leq k<n}\left|\operatorname{sgn}\left(\operatorname{sgn}\left(i_{k+1}\right)-\operatorname{sgn}\left(i_{k}\right)\right)\right| .
$$

For example, the interval pattern $[2,0,2,1,-1]$ has interval directions $[1,0,1,1,-1]$, so there is a change from ascending motion to repetition, followed by an ascending motion of two intervals, and another direction change to descending motion, yielding a total of three direction changes. Using the formula, we have $\{|\operatorname{sgn}(0-1)|,|\operatorname{sgn}(1-0)|, \mid \operatorname{sgn}(1-$ $1)|,| \operatorname{sgn}(-1-1) \mid\}=\{|-1|,|1|,|0|,|-1|\}=\{1,1,0,1\}$ where the number of direction changes is just the sum (i.e., 3). mean_run_length captures the stretches of movement in the same direction, which can be achieved by using the mean length of runs of an interval direction, where a run is defined as a subsequence of identical elements. Formally, this can be defined as the unique subset of ordered indices $j_{1}, j_{2}, \ldots, j_{m}$ with $1 \leq j_{k} \leq n$ for an n-gram $I$, such that the interval direction between these indices is constant. The index differences $r_{k}=j_{k+1}-j_{k}-1(1 \leq k<m)$ are called 'run lengths'; from this, we define the mean_run_length $(I)=1 / m \sum_{k} r_{k}$. This value is furthermore normalized to give values between 0 and 1 by dividing it by the maximal possible run length, which is $\mathrm{n}$; finally, this is subtracted from 1 to achieve the same polarity as the other measures. Hence, mean_run_length $(I)=1-$ mean_run_length $(I) / n$.

The six intrinsic measures describe different aspects of complexity. We thus calculated a combined measure for intrinsic difficulty. First, we calculated all 6 difficulty measures for all extracted n-grams. Next, we z-transformed these difficulty measures for each n-gram length separately (N-scaling) to calculate the combined sum for each n-gram instance. However, we excluded int_range and max_abs_int because they have a very broad distribution, due to occasional large intervals, and thus were numerically dominating the other measures. Finally, the sum of $\mathrm{N}$-scaled variables is again $\mathrm{N}$-scaled to ensure a standard deviation of 1 for ease of interpreting and comparing the numerical values.

\section{References}

[1] Paul F Berliner. Thinking in Jazz. 2013.

[2] J. Kathryn Bock. Toward a cognitive psychology of syntax: Information processing contributions to sentence formulation. Psychological Review, 89(1):1-47, 1982. 
[3] Matthew M. Botvinick and Jonathan D. Cohen. The computational and neural basis of cognitive control: Charted territory and new frontiers. Cognitive Science, 38(6):1249-1285, 2014 .

[4] David Brackett and Scott DeVeaux. The Birth of Bebop: A Social and Musical History. Notes, 55(1):74, 1998.

[5] Susan E Brennan. Conversation as direct manipulation: An iconoclastic view. The art of human-computer interface design, pages 393-404, 1990.

[6] Chris Cannam, Christian Landone, and Mark Sandler. Sonic visualiser: An open source application for viewing, analysing, and annotating music audio files. In MM'10 - Proceedings of the ACM Multimedia 2010 International Conference, pages 1467-1468, 2010.

[7] Charles Darwin. The Descent of Man, and Selection in Relation to Sex, volume 1-2. Princeton University Press, 2008.

[8] Stephen Grossberg. A Theory of Human Memory: Self-Organization and Performance of Sensory-Motor Codes, Maps, and Plans. In Progress in Theoretical Biology, pages 233-374. 1978.

[9] K S Lashley. The problem of serial order in behavior. In Cerebral mechanisms in behavior. Bobbs-Merrill Oxford, United Kingdom, 1951.

[10] Willem J.M. Levelt. The architecture of normal spoken language use. In Linguistic Disorders and Pathologies: An International Handbook, pages 1-15. De Gruyter Mouton, 2008.

[11] Willem J.M. Levelt, Ardi Roelofs, and Antje S. Meyer. A theory of lexical access in speech production. Behavioral and Brain Sciences, 22(1):1-75, 1999.

[12] Maryellen C. MacDonald. How language production shapes language form and comprehension. Frontiers in Psychology, 4:26, 2013.

[13] Peter F. MacNeilage and Barbara L. Davis. On the origin of internal structure of word forms. Science, 288(5465):527-531, 2000.

[14] Brian Mathias, William J Gehring, and Caroline Palmer. Electrical brain responses reveal sequential constraints on planning during music performance. Brain sciences, 9(2):25, 2019.

[15] Martin Norgaard. Descriptions of improvisational thinking by artist-level jazz musicians. Journal of Research in Music Education, 59(2):109-127, 2011.

[16] Martin Norgaard. How Jazz musicians improvise: The central role of auditory and motor patterns. Music Perception, 31(3):271-287, 2014.

[17] Martin Norgaard, Samantha N. Emerson, Kimberly Dawn, and James D. Fidlon. Creating under pressure: Effects of divided attention on the improvised output of skilled jazz pianists. Music Perception, 33(5):561-570, 2016.

[18] Martin Pfleiderer. The Weimar Jazz Database. In Martin Pfleiderer, Klaus Frieler, Jakob Abeßer, Wolf-Georg Zaddach, and Benjamin Burkhart, editors, Inside the Jazzomat. New Perspectives for Jazz Research., pages 41-84. Schott-Campus, Mainz, 2017.

[19] Jeff Pressing. Cognitive Processes in Improvisation. Advances in Psychology, 19(C):345-363, 1984.

[20] Jeff Pressing. Improvisation: Methods and models. In John A Sloboda, editor, Generative processes in music, pages 129-178. Oxford, 1988.

[21] David A. Rosenbaum, Kate M. Chapman, Matthias Weigelt, Daniel J. Weiss, and Robrecht van der Wel. Cognition, action, and object manipulation. Psychological Bulletin, 138(5):924-946, 2012. 
[22] David A. Rosenbaum, Rajal G. Cohen, Steven A. Jax, Daniel J. Weiss, and Robrecht van der Wel. The problem of serial order in behavior: Lashley's legacy. Human Movement Science, 26(4):525-554, 2007.

[23] R W Weisberg, a R Brinkman, C J Folio, a S Dick, J I Fleck, B Niederberger, and F Bassett. Toward a cognitive analysis of creativity: Improvisation in jazz. Proceedings of the Conference on Interdisciplinary Musicology Annekatrin Kessler and Frank Zimmer Graz Austria Conference on Interdisciplinary Musicology, pages 1-9, 2004. 


\title{
Spontaneous melodic productions of expert musicians contain sequencing biases seen in language production
}

\author{
Roger E. Beaty ${ }^{\mathrm{a}, 1,2}$, Klaus Frieler ${ }^{\mathrm{b}, 1}$, Martin Norgand ${ }^{\mathrm{c}}$, Hannah Merseal $^{\mathrm{a}}$, Maryellen \\ C. MACDONALD ${ }^{\mathrm{d}}$, AND DANiEl J. Weiss ${ }^{\mathrm{a}}$

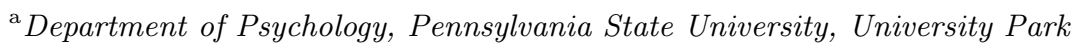 \\ ${ }^{\mathrm{b}}$ Institute for Musicology, University of Music Franz Liszt Weimar, Germany \\ ${ }^{\mathrm{c}}$ School of Music, Georgia State University, Atlanta \\ ${ }^{\mathrm{d}}$ Department of Psychology, University of Wisconsin-Madison, Madison \\ ${ }^{1}$ Authors contributed equally \\ ${ }^{2}$ Corresponding Author: Roger E. Beaty, E-mail: rebeaty@psu.edu
}

February 15, 2020

\section{SI Results}

\section{Correlations among intrinsic easiness measures.}

We examined the Pearson's correlations between the different easiness measures for each sequence length from 3 to 10. Due to the large sample size, all correlations are highly significant $(p<.001)$, so we refrain from noting p-values unless necessary. By construction, we expect large correlations between mean_int_size and int_range. Indeed, this is the case. The correlations range from $r=.91$ for shorter sequences $(N=3)$ to $r=.75$ for longer sequences $(N=10)$. Similarly, we expect strong correlations between dir_change and mean_run_length. These correlations are even higher, from $r=.95$ for $N=3$ to $r=.81$ for $N=10$. This justified the removal of one measure for each of these pairs. We retained int_range and dir_change because these have a larger variance and are somewhat easier to interpret.

We also found large negative correlations between pitch_variety and dir_change (between $r=-.50$ for $N=7$ and $r=-.46$ for $N=3$ ). This is likely because direction changes tend to lead back to a pitch that has previously occurred, particularly for sequences that predominantly consist of semitones and whole tones. Since this correlation is a meaningful result, and not due to construction, we retained both variables. There are also moderately positive correlations between int_variety and int_range (between $r=.38$ for $N=3$ and $r=.40$ for $N=5$ ), which is even larger for int_variety and mean_int_size. Notably, the correlations in the simulated corpus show similar values, so this effect seems to be a consequence of the relative rarity of large intervals. Similarly, dir_change and int_variety are also moderately correlated (between $r=.23$ for $N=3$ and $r=.41$ for $N=10$ ). This is due to the fact that a direction change implies a change of sign of subsequent intervals, which increases interval variety. As can be expected from their construction, int_variety and pitch_variety are uncorrelated (between $r=.00$ for $N=3$ and $r=.12$ for $N=10)$.

\section{Correlations between extrinsic and intrinsic easiness.}

We found that extrinsic easiness correlated positively with all measures of intrinsic easiness: int_variety $(r=.50)$, int_range $(r=.43)$, dir_change $(r=.23)$, and pitch_variety $(r=.15)$ (averaged across all N's). Simulated data revealed similar but consistently lower correlations (int_variety $r=.37$, int_range $r=.37$, pitch_variety $r=.03$ ) with the exception of direction change (dir_change $r=.26$ ). 


\section{Polynomial analysis.}

We tested whether the easiness trends are better described by polynomials and, if so, to what degree. To this end, we computed, for a set of maximum phrase positions $(10,20, \ldots, 50)$, a set of polynomial regressions of intrinsic_easiness against phrase positions for degree 1 to 5 . Analyses were computed for each N separately, and for all data ("full") and mean values per phrase position ("averaged"). We then assessed model performance by the Aikake Information Criterion (AIC) and noted all model improvements from degree $k$ to degree $k+1$, if the decrease in AIC was larger than -10 or if it was the maximum overall decrease. If more than one candidate was found, we chose the one with the largest $R_{\text {adj }}^{2}$ value. Results can be found in Fig. 4 of the main text. No linear models were chosen as the optimal model. Thus, we found an overall tendency for higher polynomial degrees for longer phrases. A bootstrap test using the poweRLaw package for $\mathrm{R}$ did not reject the null-hypothesis of a non-power law distribution $(p=.48)$, with a scaling parameter of $1.28[1,2,3]$.

\section{References}

[1] Aaron Clauset, Cosma Rohilla Shalizi, and M E J Newman. Power-law distributions in empirical data, 2009.

[2] R Development Core Team et al. R: A language and environment for statistical computing, 2011.

[3] Damiáan H Zanette. Zipf's law and the creation of musical context. Musicae Scientiae, 2006. 


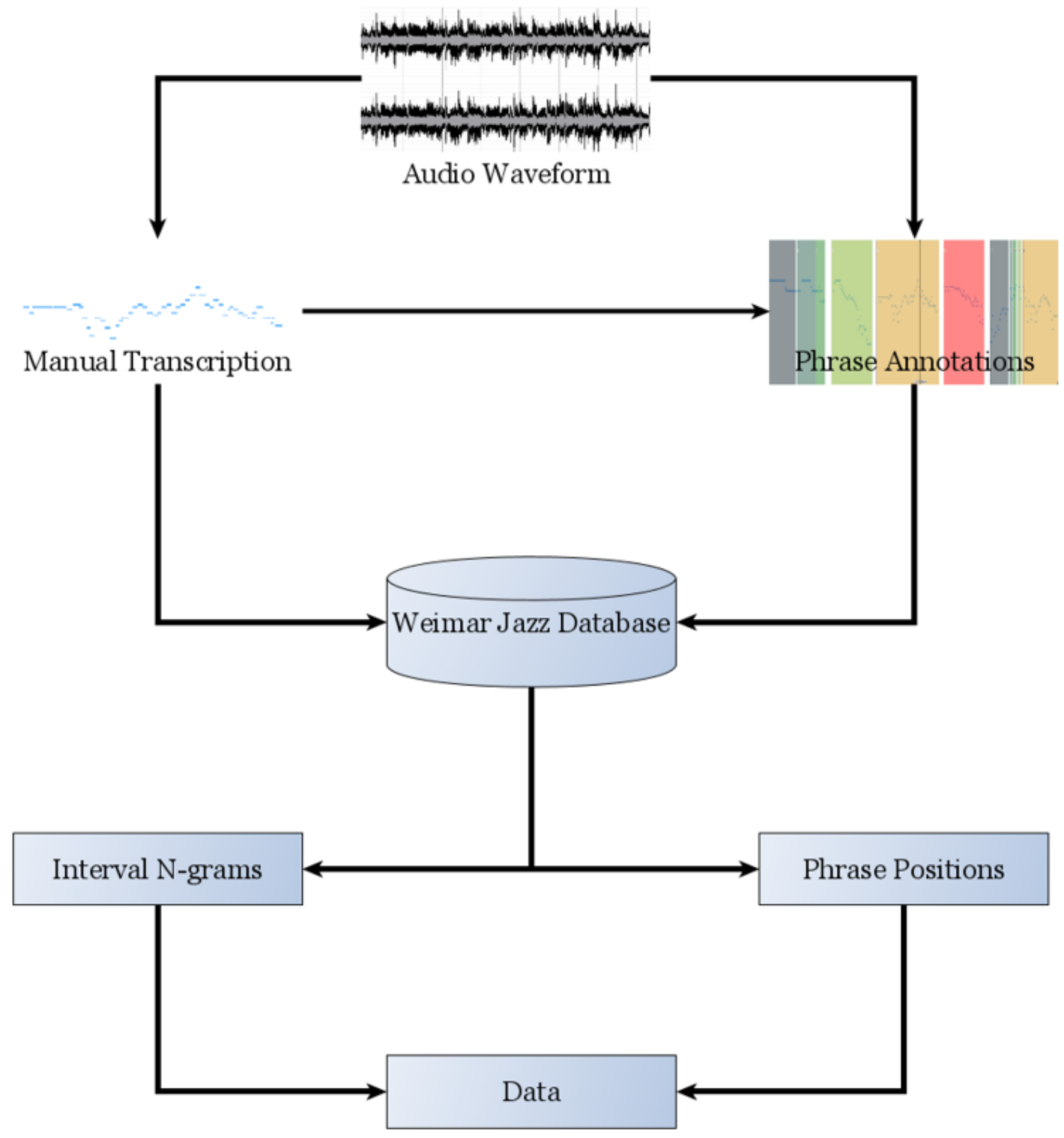

Figure 1: Flow diagram of the data assembling process. Jazz solos were manually transcribed into a piano roll -like representation and phrases were manually annotated by the transcribers. This information was collected in the Weimar Jazz Database. For the present study, we exported all interval n-grams of length 1 to 10, including their position within a solo, as well as phrase information and joined these sources in a common dataset. 